\title{
Nilotinib wirksamer als der Standard Imatinib
}

First-line Nilotinib verhinderte die Krankheitsprogression bei Patienten mit Philadelphia-Chromosom-positiver chronischer myeloischer Leukämie (Ph+ CML) in chronischer Phase besser als der Standard Imatinib. Dies bestätigte die aktuelle Auswertung der Phase-III-Studie ENESTnd nach einer medianen Nachbeobachtungszeit von etwa 18,5 Monaten (Larson R et al., ASCO 2010: \#6501).

In der Studie erhielten 846 Patienten mit neu diagnostizierter $\mathrm{Ph}+$ CML in chronischer Phase randomisiert Imatinib (400mg QD; $\mathrm{n}=283$ ) oder Nilotinib in 2 unterschiedlichen Dosierungen, der zugelassenen Standarddosierung für die Second-line-Therapie (400mg BID; $\mathrm{n}=281$ ) oder einer reduzierten Dosierung (300mg BID; $\mathrm{n}=282$ ).

Die aktuelle Auswertung ergab, dass der Anteil der Patienten mit guter molekularer Remission (MMR=Reduktion der BCR-ABLTranskripte auf $\leq 0,1 \%$ nach internationaler Skala) bzw. kompletter zytogenetischer Remission (CCyR= Philadelphia-Chromosom nicht mehr nachweisbar) unter Nilotinib auch weiterhin deutlich höher war als unter Imatinib. Die Abbildung zeigt dies am Beispiel der kumulativen MMR-Inzidenz seit der Randomisierung. Nach 12 Monaten hatte sich sowohl bei der MMR (primärer Endpunkt) als auch bei der CCyR ein signifikanter Unterschied zugunsten der First-lineTherapie mit Nilotinib gezeigt: Die MMR-Rate hatte nach 12 Monaten unter Nilotinib 44\% (300mg BID) bzw. 43\% (400mg BID) betragen (versus 22\% unter Imatinib; jeweils $\mathrm{p}<0,0001$ ), die CCyR-Rate entsprechend 80\% bzw. 78\% (versus 65\% unter Imatinib; $p<0,0001$ bzw. $p=0,0005)$.

Eine Krankheitsprogression in die akzelerierte Phase oder Blastenkrise wurde nach 18 Monaten unter Nilotinib nur bei 2 (300mg BID) bzw. 1 Patienten (400mg BID) beobachtet, unter Imatinib dagegen bei 12 Patienten ( $p=0,006$ bzw. $p=0,003$ ). Im Vergleich zu den 12-Monats-Daten der Studie ist damit nur 1 Patient mit Krankheitsprogression im Imatinib-Studienarm hinzugekommen, so Richard A. Larson, Chicago/USA.

Larson sprach sich aufgrund der im Vergleich zu Imatinib besseren Wirksamkeit von Nilotinib und einer insgesamt guten Verträglichkeit für first-line Nilotinib als neue Standardtherapie bei Patienten

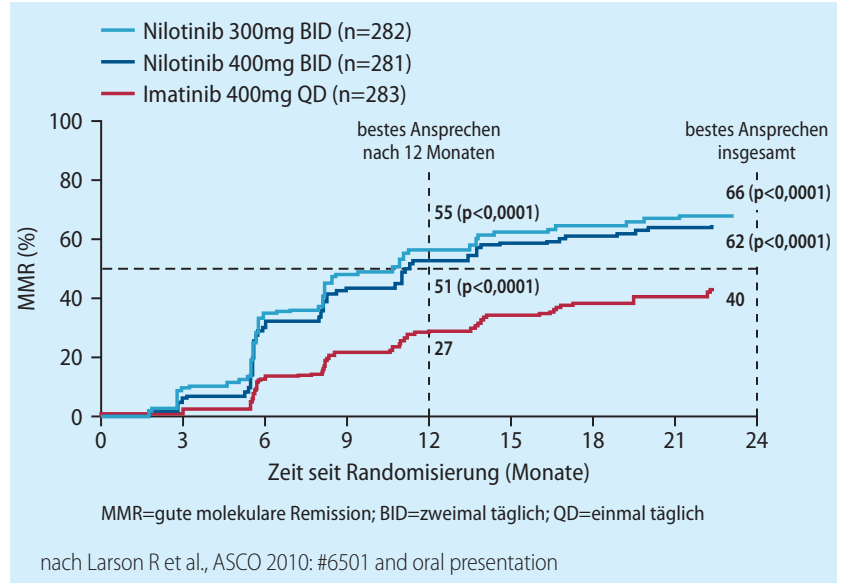

ENESTnd-Studie (Evaluating Nilotinib Efficacy and Safety in Clinical Trials of Newly Diagnosed Ph+CML Patients) - kumulative MMR-Inzidenz

mit neu diagnostizierter CML aus. Michael J. Mauro, Portland/USA, empfahl in der Diskussion der Studienergebnisse, die First-line-Zulassung der Tyrosinkinasehemmer der zweiten Generation wie Nilotinib abzuwarten und bis dahin am bisherigen Standard Imatinib festzuhalten. Bei entsprechender Zulassung empfahl Mauro den Front-line-Einsatz dieser Substanzen, wobei jedoch Patienten mit optimalem Ansprechen auf Imatinib ohne Toxizitätsprobleme nicht umgestellt, sondern weiterhin mit Imatinib behandelt werden sollten. In den USA wurde die Zulassung für first-line Nilotinib (empfohlene Dosierung 300mg BID) inzwischen in einem beschleunigten Zulassungsverfahren erteilt. Die Entscheidung der europäischen Arzneimittelbehörde steht noch aus. $A M$

\section{Fortgeschrittenes nichtkleinzelliges Lungenkarzinom}

\section{Ältere Patienten profitieren von Paclitaxel/Carboplatin}

In der Phase-III-Studie IFCT (Intergroupe Francophone de Cancérologie Thoracique) 0501 konnte nach median 21,3 Monaten Follow-up bei älteren Patienten mit fortgeschrittenem nichtkleinzelligem Lungenkarzinom (NSCLC Stadium III [ohne Option für eine Radiotherapie]/IV) unter Paclitaxel/Carboplatin ein Überlebensvorteil im Vergleich zu einer Standardmonotherapie gezeigt werden (Quoix E et al., ASCO 2010: \#2).
Im Vergleich zur Monotherapie mit Gemcitabin oder Vinorelbin wurde das mediane Gesamtüberleben (primärer Endpunkt) mit der Kombination Paclitaxel/Carboplatin von 6,2 Monaten auf 10,3 Monate signifikant verlängert ( $p=0,00004)$. Die Toxizität der Therapie war laut Quoix handhabbar.

In der Studie wurden 451 Patienten randomisiert für 4 Zyklen Paclitaxel $\left(90 \mathrm{mg} / \mathrm{m}^{2}\right.$ KOF an Tag 1, 8, 15) plus Carboplatin (AUC [Area under the Curve] 6 alle 4 Wochen) oder
5 Zyklen 3-wöchentlich an Tag 1 und 8 Gemcitabin $\left(1.150 \mathrm{mg} / \mathrm{m}^{2} \mathrm{KOF}\right)$ oder Vinorelbin (30mg/m² KOF). Second-line wurde bei Toxizität oder Progression Erlotinib (150mg/d) gegeben. Die Patienten waren zwischen 70 und 89 Jahre alt und systemisch nicht vorbehandelt. Sie hatten einen Eastern Cooperative Oncology Performance Status von 0 bis 1 (Paclitaxel/Carboplatin: 72,9\%; Kontrollgruppe: 73\%) oder 2 (Paclitaxel/Carboplatin: 27,1\%; Kontrollgruppe: $27 \%$ ). AM 